\title{
Persistent optical nuclear spin narrowing in a singly charged InAs quantum dot
}

\author{
Bo Sun, ${ }^{1}$ Wang Yao, ${ }^{2}$ Xiaodong Xu, ${ }^{3}$ Allan S. Bracker, ${ }^{4}$ Daniel Gammon,,${ }^{4}$ L. J. Sham, ${ }^{5}$ and Duncan Steel ${ }^{1, *}$ \\ ${ }^{1}$ The H. M. Randall Laboratory of Physics, The University of Michigan, Ann Arbor, Michigan 48109, USA \\ ${ }^{2}$ Department of Physics and Center of Theoretical and Computational Physics, \\ The University of Hong Kong, Hong Kong, China \\ ${ }^{3}$ Department of Physics, University of Washington, Seattle, Washington 98195, USA \\ ${ }^{4}$ Naval Research Laboratory, Washington D.C. 20375, USA \\ ${ }^{5}$ Department of Physics, The University of California San Diego, La Jolla, California 92093, USA \\ ${ }^{*}$ Corresponding author: dst@eecs.umich.edu
}

Received October 17, 2011; accepted November 23, 2011;

posted December 12, 2011 (Doc. ID 156686); published February 1, 2012

\begin{abstract}
We review the investigation of the hole-assisted dynamical nuclear spin polarization mechanism in a singly charged InAs quantum dot. Using coherent dark state spectroscopy, we measure the locking of the Overhauser field to a value determined only by the laser frequencies. Importantly, we review data that the locking effect can suppress nuclear spin fluctuations. We determine the onset time of the nuclear spin narrowing effect and its persistence absent laser interactions by directly measuring the enhancement of the electron spin coherence. This nuclear field locking effect can be explained in terms of an anisotropic hyperfine coupling between the hole spin and the nuclear spins. (C) 2012 Optical Society of America
\end{abstract} OCIS codes: $\quad 300.6250,270.1670$.

\section{INTRODUCTION}

Self-assembled quantum dots (QDs) are semiconductor structures, which confine the electron and hole wave functions in a small volume, giving rise to a discrete energy spectrum and suppressing the many-body interactions present in bulk and higher-dimensional semiconductor heterostructures [1,2] The optical transitions of a neutral or singly charged QD exhibit many classic atomic physics phenomenon such as Mollow spectra [3-ㄷ], the Autler-Townes [3] , and AC Stark effects [6,7], and experiments have demonstrated the coherent control of the spin of an electron trapped in a single QD [8]

However, unlike a single atom, a single QD has $10^{4}-10^{5} \mathrm{nu}-$ clear spins, belonging to its constituent atoms. An electron or hole trapped in a $\mathrm{QD}$ couples to these nuclei via the hyperfine interaction. The nuclear spins create an average magnetic field known as the Overhauser field [9], whose fluctuations are the major source of electron spin dephasing. The interactions of the Overhauser field with both electron and hole spins trapped in a QD has been the subject of intense study and there has been considerable interest in manipulating the nuclear spin ensemble [10-20].

In this paper, we review our recent work, which uses dark state spectroscopy to demonstrate a hyperfine interaction between the nuclear spins and an optically generated hole spin in a self-assembled QD charged with a single electron. This interaction creates a feedback mechanism that locks the Overhauser field to a value determined by the probe laser frequency [21]. The locking gives rise to hysteretic effects in the probe absorption spectrum of the $\mathrm{QD}$ and can narrow the statistical distribution of nuclear spins, suppressing the fluctuations of the Overhauser field and dramatically increasing the electron spin coherence time by up to two orders of magnitude. We measure the onset time of the nuclear spin nar- rowing (NSN) effect to be $7 \pm 1 \mathrm{~ms}$. The NSN effect can also persist for greater than $1.25 \mathrm{~s}$ after the lasers have been shut off and appears to be insensitive to fluctuations in the electron spin polarization and charge state of the QD.

Our sample is an InAs self-assembled QD embedded in a Schottky diode structure. A metal aperture mask on the sample surface gives us the spatial resolution to study only a single QD. We apply a DC bias voltage across the sample to charge the dot with a single electron. Stark shift modulation spectroscopy is used to measure the absorption spectrum via a lock-in amplifier by adding a large AC component (0.08VAC) at $3.5 \mathrm{Khz}$ to the DC voltage [22]. The inset of Fig. 1a shows the energy level diagram of the dot after a $1.32 \mathrm{~T}$ magnetic field is applied in the Voigt profile (perpendicular to the growth axis). The magnetic field axis is defined as the $\hat{x}$ axis while the growth direction is defined as the $\hat{z}$ axis. The spin ground states are labeled $|X \pm\rangle$ and the optically excited states are trion states (formed by two spin paired electrons and a hole) labeled $|T \pm\rangle$.

We selectively excite a three-level lambda $(\Lambda)$ subsystem [Fig. 1a main figure] with narrow linewidth cw lasers. When the pump and probe lasers match the two-photon Raman resonance (TPR), the lasers' coherence is imparted to the electron spin ground states. A coherent superposition of the electron spin states, known as the dark state, is formed and appears as a transparent dip in the probe absorption spectrum [23]. The depth of the dip is proportional to the electron spin decoherence rate $\left(\gamma_{s} \equiv 1 / T 2 *\right)$, making it a sensitive probe of nuclear spin fluctuations.

The probe absorption spectrum is shown in Fig. 1b where the black data is taken by scanning the probe laser from lower energy to high energy (forward) and the red data with the laser scanning from high energy to low energy (backward). 
(a)

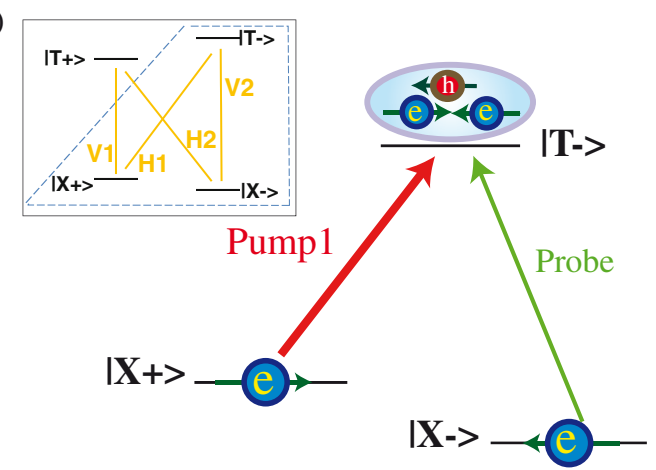

(b)

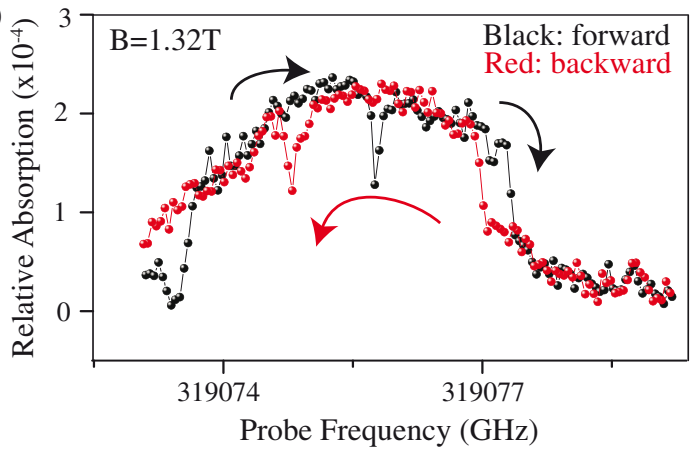

(c) Black: forward Time Constant (ms)
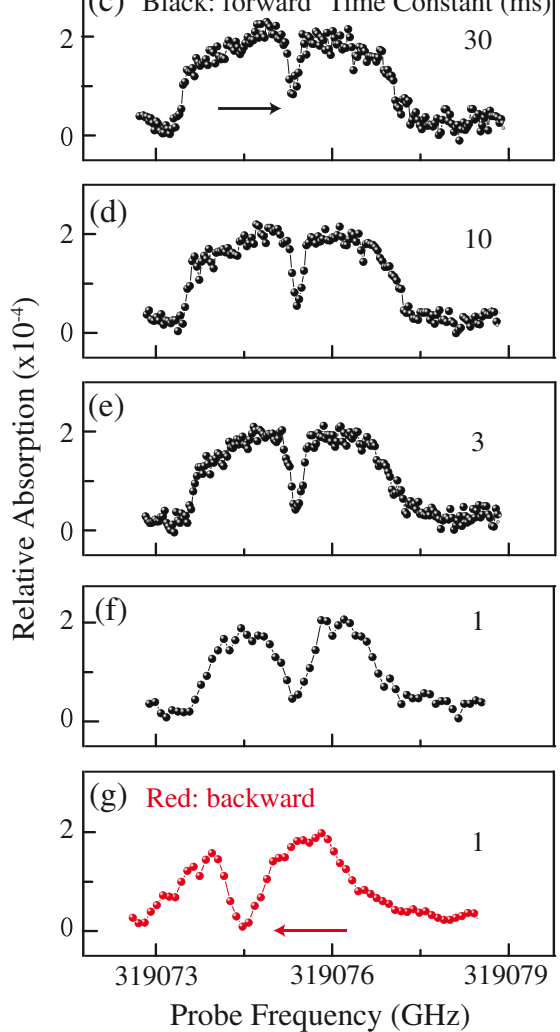

Fig. 1. (Color online) (a) (Inset) Four-level energy structure of the QD with applied magnetic field of $1.32 \mathrm{~T}$ in the Voigt geometry. (Main figure) Pump and probe lasers excite a three-level lambda $(\Lambda)$ subsystem. (b) Probe absorption spectrum for both forward and backward scans. The V2 peak is distorted from the normal dark state lineshape. Hysteresis appears at both the trailing edge and at the dark state dip. (c)-(g) Probe absorption spectra for various scan rates. Longer lock-in time constants denote slower scan rates. (g) Hysteresis occurs even at the fastest scan rate, with a $1 \mathrm{~ms}$ lock-in time constant.

Ideally, the line shape of transition V2 should be Lorentzian like with a dip (the signature of the dark state) centered on the transition [23]. The probe absorption spectrum in Fig. 1b is clearly distorted, showing a broadened line shape with a round top and sharp edges. The dark state dip is also narrower and shallower than expected. More remarkably, we observe hysteresis between the forward and backward scans at both the sharp edges of the absorption peak and the position of the dark state. The change in the position of the dark state indicates a change of the TPR when the scan direction is switched. The TPR is equal to the electron spin Zeeman splitting and is governed by the magnetic field along the $x$ axis. As the external magnetic field is fixed, these observations indicate that we optically create a dynamical nuclear spin polarization (DNP), generating an Overhauser field that depends on the laser scan direction.

We plot the absorption spectrum for different laser scan rates in Fig. 1c-g. The scan rate is given in terms of the lock-in amplifier time constant, where a short (longer) time constant indicates a faster (slower) laser scan rate. The dark state becomes more pronounced and broader as we increase the laser scan rate (i.e., the probe laser frequency is held for a shorter time at each value). With faster scan speeds, the measured line shape is closer to the standard dark state spectrum. However even at the fast scan rate with a lock-in time constant of $1 \mathrm{~ms}$, there is still a pronounced hysteresis between the forward [Fig. 1f] and backward [Fig. 1g] scans. These anomalous spectral features and their scan-rate dependence indicate the control of the Overhauser field as the probe laser frequency is scanned on a time scale comparable to the nuclear spin relaxation time (order of $1 \mathrm{~s}[\underline{15}, \underline{24}, \underline{25}]$ ). For slower scan rates, the nuclear configuration changes with laser frequency, leading to alterations of the absorption line shape.

This paper is organized in the following way. First, we present a theory of dynamic nuclear polarization through the hyperfine interaction with the optically excited hole spin. We then discuss the experimental observations and show that they can be well explained by our theoretical model. Finally, we present further experimental observations showing that the hole-assisted DNP can suppress nuclear spin fluctuations and generate a persistent NSN.

\section{THEORY}

The electron-nuclear hyperfine interaction Hamiltonian has the form [26],

$$
H_{n}=\frac{-\mu_{0}}{4 \pi} \gamma_{N} \gamma_{e} \sum_{k} \hat{\mathbf{I}}_{k} \cdot\left[\frac{8 \pi}{3} \hat{\mathbf{S}} \delta\left(\mathbf{r}_{k}\right)-\frac{r_{k}^{2} \hat{\mathbf{S}}-3 \mathbf{r}_{k}\left(\hat{\mathbf{S}} \cdot \mathbf{r}_{k}\right)}{r_{k}^{5}}+\frac{\hat{\mathbf{L}}}{r_{k}{ }^{3}}\right],
$$

where $\gamma_{N}$ and $\gamma_{e}$, respectively, are the nuclear, electron, and hole gyromagnetic ratios, and $\mathbf{r}_{\mathbf{k}}$ is the position of the electron from the $k$ th nuclear spin. $\hat{\mathbf{S}}$ and $\hat{\mathbf{L}}$ are the spin and orbital angular momentum operators of the electron and $\hat{\mathbf{I}}_{k}$ is the spin of the $k$ th nucleus. For electrons with $s$-like Bloch wave functions, the Fermi contact interaction, $\hat{\mathbf{I}} \cdot \hat{\mathbf{S}}$ dominates over the other terms. The Fermi contact hyperfine interaction between and electron and nuclear spin leads to a spin flip term, 
$S^{+} I_{k}{ }^{-}+S^{-} I_{k}{ }^{+}$, which flips a nuclear spin and an electron at the same time. In other systems, this electron mediated nuclear spin flip mechanism is cited as the cause of DNP and plays a large role in electron spin dynamics [10,15-18]. However, this interaction does not explain the phenomena seen in the experiments here.

The nuclear Zeeman energy is in the order of $10 \mathrm{~s}$ of $\mathrm{MHz}$ for a $1 \mathrm{~T}$ external magnetic field while the electron Zeeman energy is about $7 \mathrm{GHz}$. For the electron mediated spin flip to contribute to the nuclear spin dynamics in our system, the large energy mismatch must be compensated by phonons. This process is slow at cryogenic temperatures [14,27]. Moreover, the thermal energy, $k_{B} T$, is greater than the electron Zeeman splitting at $5 \mathrm{~K}$, so the phonon-assisted process is equally efficient at flipping the electron up or down and leads to a background nuclear spin polarization that is proportional to the electron spin polarization [21]. This interaction does not account for the hysteresis seen in Fig. $1 \mathrm{~b}$.

Instead, we look to the hole to explain the altered line shape and the hysteresis effects. The hole's $p$-like central cell wave function excludes the Fermi contact hyperfine, but allows the dipole-dipole interaction (second term in Eq. 1). For the QD under study, there is significant heavy hole light hole mixing as well as spin-orbit coupling so that we can define the hole as a pseudospin $1 / 2$ system where $\left|S_{h}^{z}=1 / 2\right\rangle \equiv\left|J_{z}=3 / 2\right\rangle-$ $\eta\left|J_{z}=-1 / 2\right\rangle$ and $\left|S_{h}^{z}=-1 / 2\right\rangle \equiv\left|J_{z}=-3 / 2\right\rangle-\eta\left|J_{z}=1 / 2\right\rangle$. The amount of hole mixing, $\eta$, about 0.2 in this dot, is measured using polarization dependant spectroscopy. The $z$ axis is taken to be the growth axis. If the envelope wave function varies slowly in the scale of one unit cell, the hole nuclear hyperfine Hamiltonian reduces to [21]

$$
\begin{gathered}
H_{h-n}=\sum_{k} A_{h, k}\left(S^{z} I_{k}^{z}+O(\eta)\left(S^{y} I_{k}^{y}\right)+O\left(\eta^{2}\right)\left(S^{x} I_{k}^{z}+S^{y} I_{k}^{z}\right)\right), \\
A_{h, k}=A_{h, \alpha_{k}}\left\|f_{h}\left(\mathbf{R}_{k}\right)\right\|^{2} \frac{c_{0}^{3}}{4},
\end{gathered}
$$

where $f_{h}$ is the hole envelope wave function, $A_{h}$ is the hyperfine coupling constant, and $c_{0}$ is the lattice parameter.

Since the external magnetic field is in the $\hat{x}$ direction, we define the nuclear raising and lowering operators as $I_{k}^{ \pm}=I_{k}^{y} \pm I_{k}^{z}$, resulting in a nuclear spin flip operator $S^{x} I^{ \pm}$, which can flip the nuclear spin without flipping the hole spin. This costs only the nuclear Zeeman energy, which is on the order of $10 \mathrm{MHz} / \mathrm{Tesla}$. This is smaller than the homogeneous linewidth of the trion state and energy can be conserved without the need to invoke phonon-assisted processes.

Because the laser scan rate and the dynamics of the nuclear spins is orders of magnitude slower than the optical interactions that drive the $\Lambda$ system, the three-level system is always in an instantaneous steady state, determined by the instantaneous laser frequencies and the Overhauser field. $\left|\psi_{i}\right\rangle$ and $\left|\psi_{f}\right\rangle$ denote the initial and final states of the trion system before and after a nuclear spin flip. The flip rate for the $k$ th nuclear spin due to the $O\left(\eta^{2}\right) S_{h}^{x} I_{k}^{ \pm}$interaction can be calculated using Fermi's golden rule [21]:

$$
r_{k}^{ \pm} \approx 2 \pi\left|\left\langle\psi_{f}\left|O\left(\eta^{2}\right) A_{h, k} S_{h}^{x} I_{k}{ }^{ \pm}\right| \psi_{i}\right\rangle\right|^{2} D\left( \pm \hbar \omega_{N}\right),
$$

where $r^{+}\left(r^{-}\right)$is the nuclear spin flip up (down) rate and $D\left( \pm \hbar \omega_{N}\right)$ is the density of the final states with nuclear Zeeman energy $\hbar \omega_{N}$. The total wave function is split into a direct product of the electronic state $\left|\psi^{e}\right\rangle$ and a nuclear state $\left|\psi^{N}\right\rangle$ and the spin operator $S_{h}^{x}$ only operates on the hole component so that Eq. $\underline{4}$ reduces to

$$
r_{k}^{ \pm} \approx \frac{\pi}{2} \rho_{t, i} \rho_{t, f} O\left(\eta^{4}\right) A_{h}, k^{2}\left(j \pm m_{k}\right)\left(j \mp m_{k}+1\right) D\left( \pm \hbar \omega_{N}\right),
$$

where $\rho_{t, i}\left(\rho_{t, f}\right)$ is the initial (final) trion population and $m_{k}$ is the $\hat{x}$ component of the $k$ th nuclear spin in the initial state. $j=$ $3 / 2$ for As and $9 / 2$ for In. Equation 5 shows that the spin flip rate is proportional to the product of the initial and final trion populations, $\rho_{t, i} \rho_{t, f}$. However, because the initial population $\left(\rho_{t, i}\right)$ is the same for both the flip up and the flip down process, it is clear that the faster process is one that results in a larger trion population, $\rho_{t, f}$. In other words, the hole-assisted DNP process acts like a feedback mechanism to maximize the trion population.

To work more easily with the optical Bloch equations, the DNP rate equation is converted to an equation of motion for the Overhauser shift [21], $\Delta$. We define the change in the electron Zeeman energy due to a flip of the $k$ th nuclear spin as $A_{e, k}$, which is equal to the change in laser detuning, $\delta$. The final trion population after a nuclear spin flip can be written as $\rho_{t, f}=\rho_{t, i}-\frac{\partial \rho t}{\partial \delta} A_{e, k}$. Because the number of nuclear spins is large, we can write the DNP rate for the $k$ th nuclear spin as

$$
\frac{d}{d t} m_{k}=-\gamma_{n} m_{k}+r_{k}^{+}-r_{k}^{-},
$$

where $\gamma_{n}$ is the nuclear relaxation rate and

$$
r_{k}^{+}-r_{k}^{-} \approx \pi \rho_{t} A_{h, k}^{2} O\left(\eta^{4}\right) D\left[\frac{\partial \rho_{t}}{\partial \delta} A_{e, k}\left(j^{2}-m_{k}^{2}+j\right)+\rho_{t} m_{k}\right] .
$$

We define the Overhauser shift, $\Delta \equiv \sum_{k} A_{e, k} m_{k}$, resulting in

$$
\begin{gathered}
\frac{d \Delta}{d t}=-\gamma_{N} \Delta+\alpha \rho_{t} \frac{\partial \rho_{t}}{\partial \delta}, \\
\alpha=\pi O\left(\eta^{4}\right) D \sum_{k} A_{h, k}^{2} A_{e, k}^{2}\left(j^{2}-m_{k}^{2}+j\right),
\end{gathered}
$$

where the trion population, $\rho_{t}$, is a function of the laser detuning, $\delta$, which is itself a function of the Overhauser shift, $\delta=\delta(\Delta) \cdot \gamma_{N}$ is the nuclear spin relaxation rate and $\alpha$ is a hyperfine coupling constant. The $\rho_{t} m_{k}$ term in Eq. 7 can be neglected in this case, since the total nuclear spin polarization is much less than $10 \%$.

For a strong pump and a weak probe, we can solve the optical Bloch equations to all orders of the pump and first order of the probe. The probe absorption is given by

$$
\alpha_{0} \frac{\chi^{2} \gamma_{s}+\gamma_{t}\left(\gamma_{s}^{2}+\left(\delta_{\text {probe }}-\delta_{\text {pump }}\right)^{2}\right)}{\chi^{4}+\left(\gamma_{t}^{2}+\delta_{\text {probe }}^{2}\right)\left(\gamma_{s}^{2}+\left(\delta_{\text {probe }}-\delta_{\text {pump }}\right)^{2}\right)+2 \chi^{2}\left(\gamma_{t} \gamma_{s}+\delta_{\text {probe }}\left(\delta_{\text {pump }}-\delta_{\text {probe }}\right)\right)},
$$


where $\gamma_{s}\left(\gamma_{t}\right)$ is the spin (trion) dephasing rate, $\chi$ is half the pump Rabi frequency $\Omega_{R}\left(\Omega_{R}=\frac{\mu E}{\hbar}\right.$, where $\mu$ is the dipole moment, $E$ is the electric field, and $\hbar$ is the reduced Planck constant), $\delta_{\text {probe }}\left(\delta_{\text {pump }}\right)$ is the probe (pump) detuning, and $\alpha_{0}$ is a constant. We incorporate the Overhauser shift generated by the hole-assisted DNP into the Bloch equation by replacing the probe detuning $\delta_{\text {probe }}=\omega_{\text {laser }}-\omega_{t}$ with $\delta_{\text {probe }}=\omega_{\text {probe }}-$ $\omega_{t}-\Delta / 2$, where $\omega_{\text {laser }}\left(\omega_{t}\right)$ is the laser (trion) frequency and $\Delta$ is the Overhauser shift of the electron, governed by the equation of motion given by Eq. 8 .

Figure 2a shows a simulated dark state spectrum in the absence of the hole-assisted feedback mechanism. The DNP rate of the given dark state spectrum, proportional to $\rho_{t} \frac{\partial \rho_{t}}{\partial \delta}$, is plotted in Fig. 2b. The flip rate is positive to the red of the trion maxima and negative to the blue. The feedback effect acts much like the restoring force of a spring, with the trion maxima (the green circles in Fig. 2b located at laser detunings of $\delta= \pm \Omega_{\text {pump }} / 2$ ) acting as stability points, where the flip rate goes to zero. Figure $2 \mathrm{c}$ simulates a slow laser scan including the DNP feedback effect. Figure $1 \mathrm{~b}$ is best reproduced using $\alpha=2.4(\mathrm{MHz})^{3}$ and $\gamma_{n}=1.5 \mathrm{~S}^{-1}$. The calculated Overhauser shift is plotted in Fig. 2d.

As the laser scans, the laser detuning is moved from the point of maximum trion excitation. The DNP feedback mechanism shifts the Overhauser field, and thus the Zeeman splittings, to compensate for the laser detuning. The trion resonance is pulled toward the laser, creating sharp transitions while broadening the absorption peaks. The Overhauser shift changes sign depending on the laser scan direction, creating a hysteresis between forward and backward scans. When the laser scans faster than the nuclei can flip, the line shape more closely resembles a normal dark state absorption spectrum. This is simulated in Figs. 2e-2f using $\alpha=50(\mathrm{MHz})^{3}$ and $\gamma_{n}=$ $2.5 \mathrm{~S}^{-1}$ to best match the experimental data in Figs. 1c-1d. A larger hysteresis loop can be generated by detuning the pump laser, shown in Fig. 2g.

\section{HOLE-ASSISTED DNP}

We can examine the dynamics of the Overhauser field at the TPR by monitoring the dark state. We first take a forward scan to locate the dark state (black curve in Fig. 3a), where the magnetic field has been increased to $2.64 \mathrm{~T}$. We then take a partial forward scan to prepare the initial nuclear spin configuration and stop the laser at point $L$, just before the formation of the dark state (red curve). Immediately, the absorption signal is measured as a function of time [Fig. 3b]. From the signal level, we can tell that the system starts in configuration $L$, jumps into the dark state (configuration $D$ ) where it stays for about $40 \mathrm{~s}$, and then switches to configuration $R$, where it remains at a high probe absorption state. In experiments, the system can stay in the dark state from a few seconds to $3 \mathrm{~min}$, indicating the metastable nature of the nuclear configuration at the TPR.
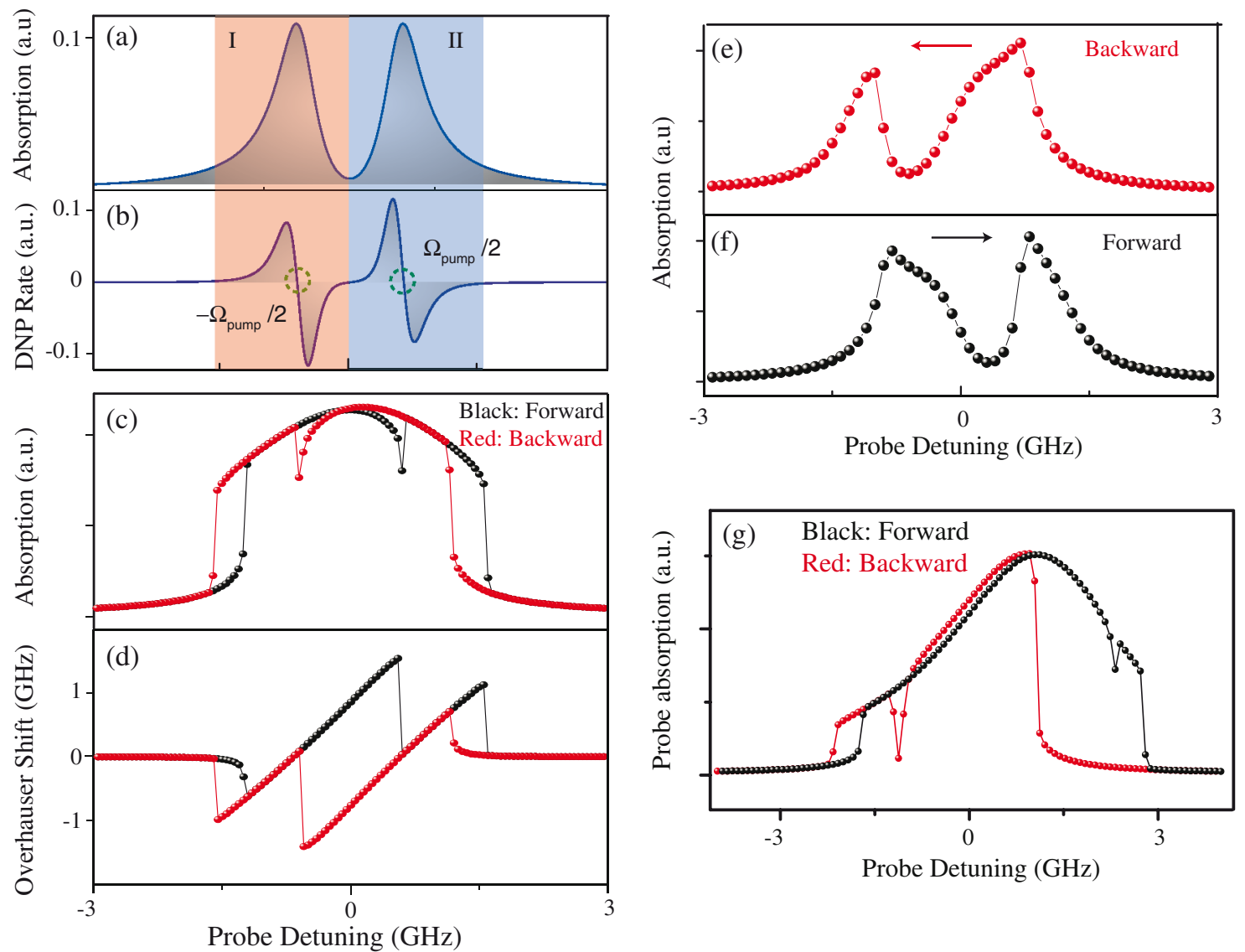

Fig. 2. (Color online) (a) Simulated dark state absorption spectrum in the absence of the hole-assisted DNP effect. (b) Plot of $\rho_{t} \frac{\partial \rho_{t}}{\partial \delta}$, which is proportional to the nuclear spin flip rate. (c) Simulated absorption spectrum for a slow laser scan including the hole-assisted DNP effect. (d) Overhauser shift experienced by the electron spin, giving rise to the spectrum in (c). (e), (f) Simulated absorption spectrum for a fast laser scan including the hole-assisted DNP effect for backward (e) and forward (f) scans. (g) Larger hysteresis loops can be generated by changing the pump detuning. 

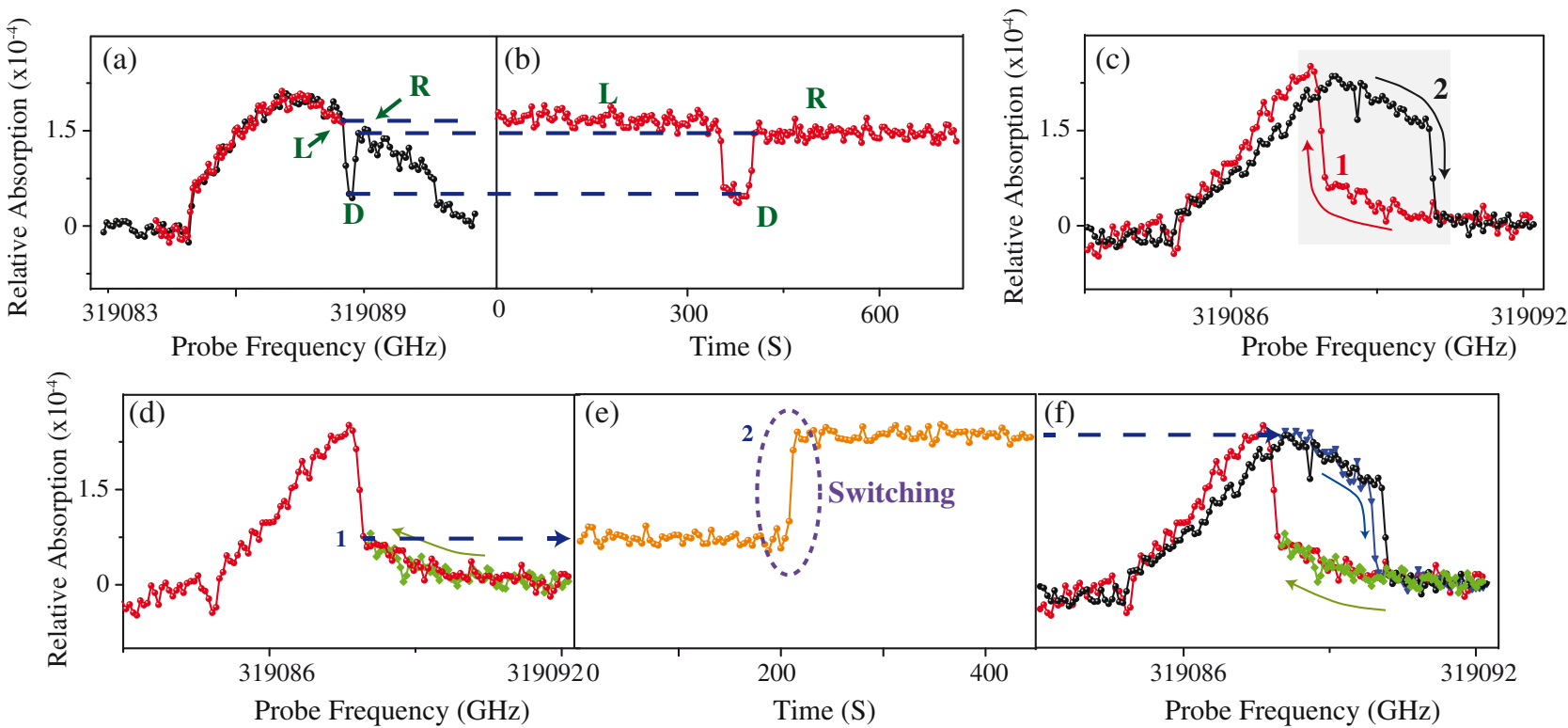

Fig. 3. (Color online) Data taken at a magnetic field of 2.64 T. (a) Black (red) curve is a full (partial) forward scan. $L, D$, and $R$ label three different system configurations. (b) Probe absorption as a function of time taken immediately after stopping the laser at $L$. (c) Black (red) curve is forward (backward) scan spectrum with the pump laser detuned to maximize the hysteresis loop. (d) Green curve is a partial backward scan. (e) Probe absorption as a function of time taken immediately after the green curve in (d). (f) Partial forward scan taken after the system switches hysteresis states.

The metastablility of the dark state can be understood by examining the DNP rate shown in Fig. 2b. The two stable regions I and II lock the probe absorption to trion maxima, the points circled in green. The TPR is exactly between these two stability regions and, because the slope is positive, is in fact an unstable point. However, the DNP rate is clearly zero at the TPR, and the slope of the DNP rate around the TPR is still small. So although the TPR is an unstable state for the DNP feedback mechanism, the dynamics at this point are slow enough as to make it metastable.

To show that the hole-assisted feedback mechanism maximizes the trion population, we make a set of measurements with the pump detuned to maximize the hysteresis loop. Figure 3c shows the probe absorption spectra with forward (black) and backward (red) scans. The probe laser is first scanned backward and stopped just before the sharp rising edge of the absorption peak, given by the green curve in Fig. 3d. The probe absorption signal is measured as a function of time with the laser frequency fixed. As we see by the time dependant absorption signal in Fig. 3e, the system remains in hysteresis state 1 for some time and then abruptly switches into hysteresis state 2 . The system switches from an unstable state to a stable one, where the trion is maximally excited. After the time-dependant data, the probe laser is scanned forward again and we find that the subsequent partial forward scan spectrum (the blue curve in Fig. 3d) overlaps significantly with the full forward scan.

\section{NUCLEAR SPIN NARROWING}

The self-locking effect observed in the spectroscopy also leads to the suppression of the nuclear spin fluctuations. Once the system has switched to a configuration of maximum trion excitation, the electron spin Zeeman energy and hence the nuclear field are locked to the instantaneous laser frequencies. In this regime, the DNP feedback actively works to maximize the trion population. If the Overhauser field fluctuates, the Zeeman splitting changes, moving the trion transition slightly out of resonance with the laser. The DNP feedback effect immediately compensates for this shift and effectively dampens nuclear spin fluctuations, generating NSN. This is illustrated in Fig. 4a. Microscopic calculations on the DNP rate by the optically excited hole showed that there is indeed a steady-state NSN when the laser frequencies are held constant at the trion excitation maximums [20]. Because nuclear fluctuations are main source of electron spin dephasing in this system, we should see an enhancement of the electron spin $T 2 *$ time in the dark state absorption spectra.

We take a series of pump power-dependent absorption spectra to measure the electron spin decoherence. Figure $4 \mathrm{~b}$ shows an example spectrum measured using fast forward scan. The electron spin decoherence rate can be estimated using two methods. We can extract the $\gamma_{s}$ from best-fit simulations that incorporate the hole-assisted DNP with the optical Bloch equations, simulating the Overhauser shift. These rates are given in Fig. 4c as the purple triangles.

Solving the optical Bloch equations for a strong pump and weak probe in the lambda system, we can find the probe absorption at the dark state dip $\left(\alpha_{\text {dip }}\right)$ and the Rabi sideband $(\alpha$ peak $)$

$$
\begin{gathered}
\alpha_{\text {dip }}=\alpha_{0} \frac{\chi^{2} \gamma_{s}+\gamma_{t}\left(\gamma_{s}^{2}\right)}{\chi^{4}+2 \chi^{2} \gamma_{t} \gamma_{s}+\gamma_{t}^{2} \gamma_{s}^{2}}, \\
\alpha_{\text {peak }}=\alpha_{0} \frac{\chi^{2} \gamma_{s}+\gamma_{t}\left(\gamma_{s}^{2}+\chi^{2}\right)}{2 \chi^{2} \gamma_{t} \gamma_{s}+\gamma_{t}^{2} \gamma_{s}^{2}+\left(\gamma_{t}^{2}+\gamma_{s}^{2}\right) \chi^{2}},
\end{gathered}
$$

where $\chi$ is half the pump 1 Rabi frequency, $\gamma_{t}$ is the trion dephasing rate, $\gamma_{s}$ is the electron spin dephasing rate, and $\alpha_{0}$ is a constant. In the limit where $\gamma_{s} \ll \chi, \gamma_{t}$, the ratio between the dip and peak absorption reduces to $\frac{\alpha_{\mathrm{dip}}}{\alpha_{\text {peak }}} \approx \frac{\gamma_{t} \gamma_{s}}{\chi^{2}}$. For this QD, we use $\gamma_{t}=400 \mathrm{MHz} \cdot \gamma_{s}$ estimated using this method is shown in 
Fig. $\underline{4 c}$ as the black dots. We estimate that the spin dephasing due to a thermally distributed nuclear environment to be $(360 \pm 30) \mathrm{MHz}$ and is given by the orange dotted line.

The suppression of spin dephasing via the hole-assisted feedback mechanism is determined by the slope of the nuclear spin flip rate as a function of detuning at the locking points, i.e., the two circled positions in Fig. $2 b$ at $\pm \Omega_{\text {pump }} / 2$. A larger slope means a stronger restoring force, and hence a better locking effect. As the pump power increases, the slope, and thus the restoring force increase as well.

For spectra in Figs. $\underline{4 b}$ and $\underline{4 c}$, the probe scans quickly to generate a clean absorption spectrum. However, since the scan rate is faster than the nuclear equilibration rate, we do not fully realize the NSN that can be generated. Figure $4 d$ shows a laser geometry using two pumps to lock the Overhauser field. Pump 1 remains near resonant with transition $\mathrm{H} 1$ and pump 2 is tuned to transition V2 and fixed at a frequency, which maximizes the trion absorption. The two pumps generate NSN while the weak probe beam measures the dark state spectrum with a fast scan rate. The probe is weak and scans at a fast rate to minimize the effect of the probe beam on the nuclear field.

The three-beam absorption spectrum is shown in Fig. 4e. It has a clean dark state line shape with a more pronounced dip compared to the two-beam spectrum in Fig. $\underline{4 b}$ at a similar pump power. The two pumps lock the nuclear field and create a larger NSN effect. The dark state dip is proportional to $\gamma_{s}$, and it is clear that the electron spin dephasing is substantially reduced. Fitting the data with the standard two beam optical Bloch equation (Eq. $\underline{10}$ using parameters $\Omega_{\text {pump }} / 2 \pi=$ $0.9 \mathrm{GHz}, \gamma_{t} / 2 \pi=0.4 \mathrm{GHz}$ and $\delta_{\text {pump }}=-30 \mathrm{MHz}$ ) yields $\gamma_{s} / 2 \pi$ on the order of $1 \mathrm{MHz}$ with a $5 \mathrm{MHz}$ upper bound error bar and the red curve. We also estimate the $T 2 *$ by using the previously described method of ratios, giving a $\gamma_{s} / 2 \pi$ of $2 \mathrm{MHz}$, which agrees with the fit.

It is possible that there is a coherent coupling between pump 2 and the probe laser which may distort the dark state line shape, leading to errors in the three-beam measurement of $\gamma_{s}$. To avoid this effect and to measure the time dynamics of the NSN with finer time resolution, we pass all three lasers through acousto-optical shutters to individually gate the lasers and separate the preparation of NSN from the readout of the electron spin coherence time.

To measure the onset time of the NSN effect we gate the lasers using the gate timing diagram in Fig. 5a. Pumps 1 and 2 are first gated on, populating the trion state and generating NSN ("initialization"). To read out the dark state, pump 2 is gated off and the probe is gated on for $25 \mathrm{~ms}$ ("readout"). The timing diagram in Fig. 5a shows the gating of the lasers for each point in a probe absorption scan.
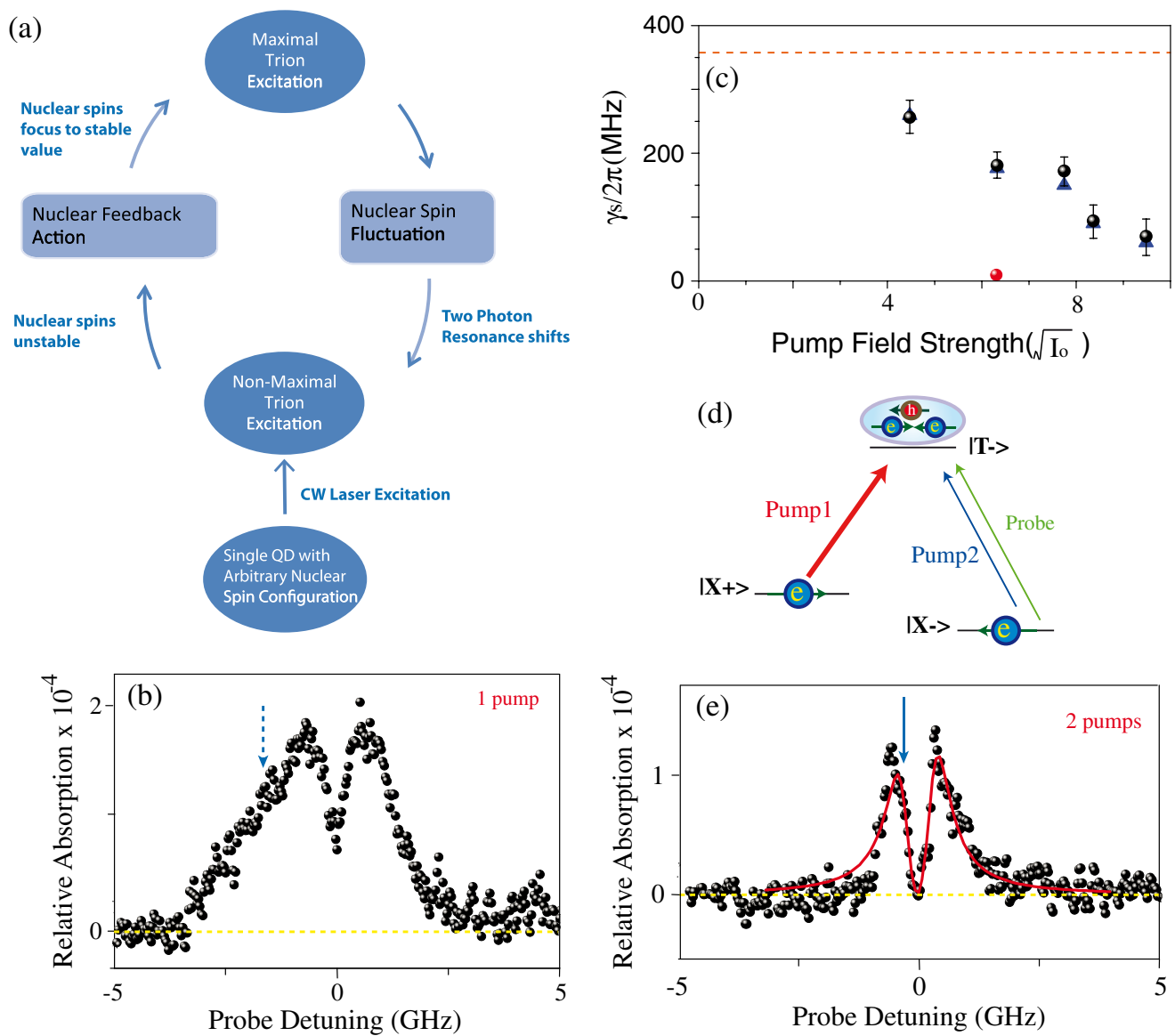

Fig. 4. (Color online) (a) Schematic of the DNP feedback mechanism. (b) Example of the spectra measured using a fast forward scan. (c) Estimated electron spin decoherence rate $\left(\frac{\gamma_{s}}{2 \pi}\right)$ from two beam absorption spectra. $I_{0}=2 \mathrm{~W} \mathrm{~cm}^{-2}$. The black dots are rates inferred from absorption peak to dark state depth ratios while the triangles are values extracted from best-fit simulations. The dashed line is the thermal value of the decoherence rate, $360 \mathrm{MHz}$. The red dot is the value from a fit of the three beam spectrum in (d). (d) Three-beam laser geometry. (e) Three-beam measurement. The red curve is a fit to the optical Bloch equations. The blue arrow indicates the position of pump 2 [also the dashed arrow in (c) for reference]. 

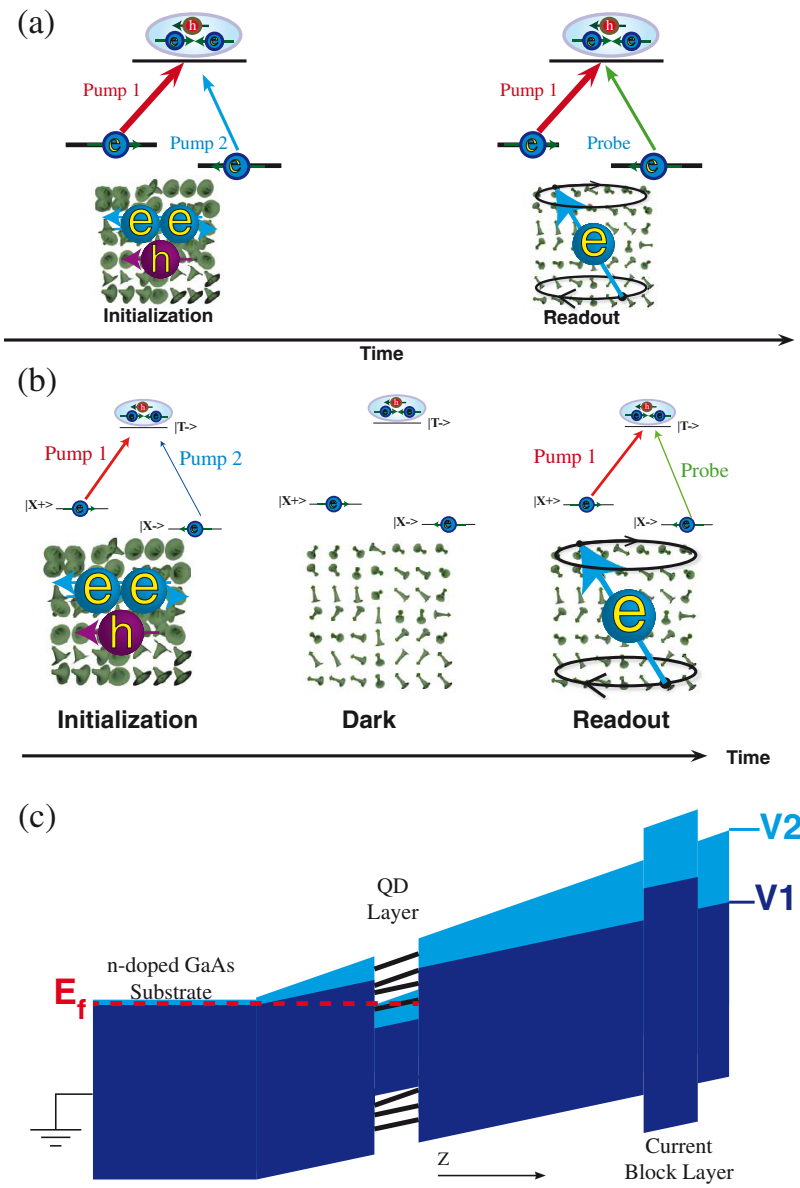

Fig. 5. (Color online) (a) Cartoon illustrating the timing of the laser gating at each point of an absorption spectrum. The nuclear spins (green arrows in the background with Gaussian envelopes) start in a state of large fluctuation. The NSN state is represented by narrower Gaussian envelopes, but has a similar average field. (b) Dark period is inserted into the gating sequence. (c) Band diagram as a function of position along the growth direction. $E_{f}$ is the Fermi level and V1 and V2 represent the peak-to-peak modulation voltages.

Preliminary data indicates that the NSN onset has a $1 / e$ time of $7 \pm 1 \mathrm{~ms}$ at $5 \mathrm{~K}$ and $12 \pm 6 \mathrm{~ms}$ at $14 \mathrm{~K}$. Because the measured onset time of the hole-assisted DNP effect is shorter than the readout time, the Overhauser field weakly locks to the probe laser frequency as it scans and distorts the measured line shapes (data not shown). As there is no hole population at the TPR, this does not affect the NSN measurement, only the average Overhauser field build up. Hence, the perturbation to the nuclei due to the probe does not impact our conclusion regarding the preparation time scales of the NSN state. We estimate that for a $100 \mathrm{~ms}$ initialization time, we have reduced the electron spin dephasing rate to $\gamma_{s} / 2 \pi=6 \mathrm{MHz}$ with an upper bound error of $14 \mathrm{MHz}$.

To measure the persistence of the NSN in absence of laser interaction, we insert a dark period between the initialization and readout stages, with the gate timing diagram given by Fig. 5b. We have seen that the NSN can persist without laser interaction for more than $1.25 \mathrm{~s}$, as indicated by the level of absorption at the TPR.

Additionally, the sample bias is being modulated according to the Stark shift modulation technique during this time. Figure $5 \mathrm{c}$ shows the band diagram of the sample as a function of the position along the growth axis ( $z$ axis). The Fermi energy $\left(E_{f}\right)$ is determined by the $n$-doped GaAs substrate. Because the modulation is large, voltages applied across the sample change the QD energies so that the electrons at the Fermi level shift in (V1) and out (V2) of resonance with dot. When the voltage is V2, the electron is shifted to an unstable point (cotunneling region [28]) between the neutral exciton and trion bias regions [29] during one-half period of the modulation cycle. The electron is randomly reinitialized at a rate of at least $3.5 \mathrm{KHz}$, corresponding to the modulation frequency. It appears that NSN is insensitive to the electron charge and spin orientation [30]. The literature indicates that the narrowed nuclear spin distribution may persist from tens of seconds up to an hour $[\underline{15}, \underline{24}]$.

\section{CONCLUSION}

In summary, we review recent experiments that show that there exists a hole-assisted DNP feedback mechanism arising from the noncolinear hyperfine coupling between the hole spin and the nuclear spins in an InAs self-assembled QD. This feedback mechanism locks the Overhauser field to a value determined by the laser frequency by shifting the TPR to maximize trion excitation. This feedback mechanism can also be used to prepare the nuclear spins in a singly charged QD into a NSN state, which can persist in the dark for more than $1 \mathrm{~s}$ and has a preparation time of tens of milliseconds. The NSN depends only on the hole spin and appears insensitive to the electron charge and spin orientation.

\section{ACKNOWLEDGMENTS}

We thank L.-M. Duan and Z.-X. Gong for fruitful discussions. This research was supported by the United States Army Research Office (USARO) MURI award W911NF0910406. The authors would also like to acknowledge the National Science Foundation (NSF), United States Air Force Office of Scientific Research (AFOSR), and Defense Advanced Research Projects Agency (DARPA) for their support.

\section{REFERENCES}

1. H. Wang, K. Ferrio, D. G. Steel, Y. Z. Hu, R. Binder, and S. W. Koch, "Transient nonlinear optical response from excitation induced dephasing in GaAs," Phys. Rev. Lett. 71, 1261-1264 (1993)

2. V. M. Axt and A. Stahl, "A dynamics-controlled truncation scheme for the hierarchy of density matrices in semiconductor optics," Z. Phys. B 93, 195-204 (1994).

3. X. Xu, B. Sun, P. R. Berman, D. G. Steel, A. S. Bracker, D. Gammon, and L. J. Sham, "Coherent optical spectroscopy of a strongly driven quantum dot," Science 317, 929-932 (2007).

4. R. Melet, V. Voliotis, A. Enderlin, D. Roditchev, X. L. Wang, T. Guillet, and R. Grousson, "Resonant excitonic emission of a single quantum dot in the rabi regime," Phys. Rev. B 78, 073301 (2008).

5. E. B. Flagg, A. Muller, J. W. Robertson, S. Founta, D. G. Deppe, M. Xiao, W. Ma, G. J. Salamo, and C. K. Shih, "Resonantly driven coherent oscillations in a solid-state quantum emitter," Nat. Phys. 5, 203-207 (2009).

6. X. Xu, B. Sun, E. D. Kim, K. Smirl, P. R. Berman, D. G. Steel, A. S. Bracker, D. Gammon, and L. J. Sham, "Single charged quantum dot in a strong optical field: absorption, gain, and the ac-Stark effect," Phys. Rev. Lett. 101, 227401 (2008).

7. M. Kroner, C. Lux, S. Seidl, A. W. Holleitner, K. Karrai, A. Badolato, P. M. Petroff, and R. J. Warburton, "Rabi splitting and ac-Stark shift of a charged exciton," Appl. Phys. Lett. 92, 031108 (2008). 
8. D. Press, T. D. Ladd, B. Zhang, and Y. Yamamoto, "Complete quantum control of a single quantum dot spin using ultrafast optical pulses," Nature 456, 218-221 (2008).

9. I. A. Merkulov, A. L. Efros, and M. Rosen, "Electron spin relaxation by nuclei in semiconductor quantum dots," Phys. Rev. B 65, 205309 (2002).

10. D. Stepanenko, G. Burkard, G. Giedke, and A. Imamoglu, "Enhancement of electron spin coherence by optical preparation of nuclear spins," Phys. Rev. Lett. 96, 136401 (2006).

11. G. Giedke, J. M. Taylor, D. D' Alessandro, M. D. Lukin, and A. Imamoglu, "Quantum measurement of a mesoscopic spin ensemble," Phys. Rev. A 74, 032316 (2006).

12. D. Klauser, W. A. Coish, and D. Loss, "Nuclear spin state narrowing via gate-controlled rabi oscillations in a double quantum dot," Phys. Rev. B 73, 205302 (2006).

13. M. S. Rudner and L. S. Levitov, "Self-polarization and dynamical cooling of nuclear spins in double quantum dots," Phys. Rev. Lett. 99, 036602 (2007).

14. J. Danon and Y. V. Nazarov, "Nuclear tuning and detuning of the electron spin resonance in a quantum dot: theoretical consideration," Phys. Rev. Lett. 100, 056603 (2008).

15. D. J. Reilly, J. M. Taylor, J. R. Petta, C. M. Marcus, M. P. Hanson, and A. C. Gossard, "Suppressing spin qubit dephasing by nuclear state preparation," Science 321, 817-821 (2008).

16. I. T. Vink, K. C. Nowack, F. H. L. Koppens, J. Danon, Y. V. Nazarov, and L. M. K. Vandersypen, "Locking electron spins into magnetic resonance by electron-nuclear feedback," Nat. Phys. 5, 764-768 (2009).

17. C. Latta, A. Hogele, Y. Zhao, A. N. Vamivakas, P. Maletinsky, M. Kroner, J. Dreiser, I. Carusotto, A. Badolato, D. Schuh, W. Wegscheider, M. Atature, and A. Imamoglu, "Confluence of resonant laser excitation and bidirectional quantum-dot nuclear-spin polarization," Nat. Phys. 5, 758-763 (2009).

18. H. Bluhm, S. Foletti, D. Mahalu, V. Umansky, and A. Yacoby, "Enhancing the coherence of a spin qubit by operating it as a feedback loop that controls its nuclear spin bath," Phys. Rev. Lett. 105, 216803 (2010).
19. W. A. Coish, J. Fischer, and D. Loss, "Free-induction decay and envelope modulations in a narrowed nuclear spin bath," Phys. Rev. B 81, 165315 (2010).

20. W. Yang and L. J. Sham, "Collective nuclear stabilization by optically excited hole in quantum dot," arXiv: 1012.0060v2 (2010).

21. X. Xu, W. Yao, B. Sun, D. G. Steel, A. S. Bracker, D. Gammon, and L. J. Sham, "Optically controlled locking of the nuclear field via coherent dark-state spectroscopy," Nature 459, 1105-1109 (2009).

22. B. Alen, F. Bickel, K. Karrai, R. J. Warburton, and P. M. Petroff, "Stark-shift modulation absorption spectroscopy of single quantum dots," Appl. Phys. Lett. 83, 2235-2237 (2003).

23. X. Xu, B. Sun, P. R. Berman, D. G. Steel, A. S. Bracker, D. Gammon, and L. J. Sham, "Coherent population trapping of an electron spin in a single negatively charged quantum dot," Nat. Phys. 4, 692-695 (2008).

24. A. Greilich, A. Shabaev, D. R. Yakovlev, A. L. Efros, I. A. Yugova, D. Reuter, A. D. Wieck, and M. Bayer, "Nuclei-induced frequency focusing of electron spin coherence," Science 317, 1896-1899 (2007).

25. P. Maletinsky, C. W. Lai, A. Badolato, and A. Imamoglu, "Nonlinear dynamics of quantum dot nuclear spins," Phys. Rev. B 75 035409 (2007).

26. A. Abragam, The Principles of Nuclear Magnetism (Claredon, Oxford, 1973).

27. A. Khaetskii and Y. Nazarov, "Spin-flip transitions between zeeman sublevels in semiconductor quantum dots," Phys. Rev. B 64125316 (2001).

28. M. Atature, J. Dreiser, A. Badolato, A. Hogele, K. Karrai, and A. Imamoglu, "Quantum-dot spin-state preparation with near-unity fidelity," Science 312, 551-553 (2006).

29. R. J. Warburton, C. Schaflein, D. Haft, F. Bickel, A. Lorke, K. Karrai, J. M. Garcia, W. Schoenfeld, and P. M. Petroff, "Optical emission from a charge-tunable quantum ring," Nature 405, 926929 (2000).

30. Z. Gong, Z.-Q. Yin, and L. Duan, "Dynamics of the Overhauser field under nuclear spin diffusion in a quantum dot," New J. Phys. 13, 033036 (2011). 